\title{
Frecuencia y factores asociados al consumo de alcohol, cigarrillo y sustancias psicoactivas, en estudiantes de una Universidad Pública en Colombia: un estudio mixto
}

Frequency and factors associated to the use of, alcohol, smoking and psychoactive substances, in students from a Public University in Colombia: A mix-methods study

Frequência e fatores associados com o consumo de álcool, cigarro e substâncias psicoativas em estudantes de uma universidade pública colombiana: estudo misto

\author{
Alejandra González-Ospina ${ }^{1}$ \\ Andrea Rodríguez-Restrepo ${ }^{2}$ \\ María Mercedes Grajales-Román ${ }^{3}$ \\ Yeraldyn Espinosa-Chirivi ${ }^{4}$ \\ Marta L. Martínez-Gómez ${ }^{5}$ \\ Andrés A. Agudelo-Suárez ${ }^{6}$
}

Recibido: 21 de marzo de 2020

Aprobado: 3 de mayo de 2020

Publicado: 1 de junio de 2020

Cómo citar este artículo:

González-Ospina A, Rodríguez-Restrepo A, Grajales-Román MM, Espinosa-Chirivi Y, Martínez-Gómez ML, Agudelo-Suárez AA. Frecuencia y factores asociados al consumo de alcohol, cigarrillo y sustancias psicoactivas, en estudiantes de una Universidad Pública en Colombia: un estudio mixto. Revista Nac. Odontol. (2020); 16(1), 1-19. doi: https://doi.org/10.16925/2357-4607.2020.01.02

Artículo de investigación. https://doi.org/10.16925/2357-4607.2020.01.02

1 Odontóloga. Universidad de Antioquia.

ORCID: https://orcid.org/0000-0001-6202-7768

2 Odontóloga. Universidad de Antioquia.

ORCID: https://orcid.org/0000-0003-0741-9624

3 Odontóloga. Universidad de Antioquia.

ORCID: https://orcid.org/0000-0002-4487-6165

4 Odontóloga. Universidad de Antioquia.

ORCID: https://orcid.org/0000-0003-3206-1249

5 Psicóloga. Magíster en Salud Pública. Profesora Ocasional Universidad de Antioquia.

ORCID: https://orcid.org/0000-0002-0586-3193

6 Odontólogo. Especialista en Administración de Servicios de Salud. PhD en Salud Pública. Profesor Asociado. Facultad de Odontología. Universidad de Antioquia.

ORCID: https://orcid.org/0000-0002-8079-807X.

Correo electrónico: alonso.agudelo@udea.edu.co 
2 Frecuencia y factores asociados al consumo de alcohol, cigarrillo y sustancias psicoactivas, en estudiantes de una Universidad Pública en Colombia: un estudio mixto

\section{Resumen}

Introducción: establecer sistemas de apoyo social a la población universitaria hace necesario su caracterización en salud, estilos de vida y prácticas de riesgo.

Objetivo: determinar la frecuencia y los factores asociados al consumo de sustancias psicoactivas, alcohol y cigarrillo en estudiantes de pregrado de la Facultad de Odontología (Universidad de Antioquia).

Métodos: estudio mixto: (1) estudio descriptivo transversal con 162 participantes (54,3\% mujeres) mediante encuesta y análisis uni/bivariado, calculando frecuencias y pruebas Chi cuadrado. (2) Estudio cualitativo mediante 6 grupos focales, análisis narrativo de contenido y triangulación de resultados. Se garantizaron los principios éticos para la investigación en salud.

Resultados: el 30\% manifestó embriagarse en los últimos 30 días (mayor proporción en hombres: 40\% y p<0,05). La edad mediana de inicio de consumo de alcohol fue de 15,0 años (Rango Intercuartílico RIC 14,0-16,0). La frecuencia de consumo de cigarrillo actual fue $11 \%$ y la experiencia (actual/pasada) fue 15\%, en ambos casos mayor en hombres. El consumo de sustancias psicoactivas en el último año fue de $22 \%$ y la edad mediana de inicio del consumo fue de 16,0 años (RIC 15,0-18,5) (más temprana en hombres). El análisis cualitativo develó categorías relacionadas al consumo: 1) estado anímico, 2) carga académica, 3) utilización del tiempo libre, 4) relaciones interpersonales, 5) condiciones relacionadas con apreciaciones y experiencias previas frente al consumo propio y de personas de su alrededor.

Conclusiones: se encontraron factores asociados al consumo de alcohol, cigarrillo y sustancias psicoactivas que deben considerarse para el diseño de programas de promoción y prevención.

Palabras clave: consumo de bebidas alcohólicas; hábito de fumar; trastornos relacionados con sustancias; estudiantes de Odontología

\section{Abstract}

Background: to establish social support systems for university groups requires their characterization in terms of health, lifestyles and risk practices.

Objective: To determine the frequency and factors associated with the use of psychoactive substances, alcohol and smoking in undergraduate students of the Faculty of Dentistry (University of Antioquia).

Methods: Mix-methods study: 1) a descriptive study was conducted in 162 participans (54.3\% female) by means of a survey and uni/bivariate analyses were performed, calculating absolute and relative frequencies and Chi square tests. 2) a qualitative study was conducted, by means of 6 focus groups, narrative content analysis and triangulation of the results. The ethical principles for health research were guaranteed.

Results: $30 \%$ reported high alcohol drinking in the last 30 days (greater proportion in men: $40 \%$ and $p<0.05$ ). Median age for beginning alcohol consumption was 15.0 (Interquartile range IQR 14.0-16.0) years. The frequency of current smoking was $11 \%$ and the experience (current/past) was $15 \%$, in both cases greater in men. The consumption of psychoactive substances in the last year was $22 \%$ (greater in men) and the median age for beginning the consumption was 16.0 (IQR 15.0-18.5) years (earlier in men). Qualitative analysis unveiled categories related to consumption: 1) mood, 2) academic load, 3) profile of free time, 4) interpersonal relationships, 5) conditions related to opinions and previous experiences of consumption of themselves and of people around them.

Conclusions: Associated factors to the consumption of psychoactive substances, alcohol and smoking were observed that should be considered for designing promotion and prevention programs.

Keywords: Alcohol Drinking; Smoking; Substance-Related Disorders; Students, Dental. 
Alejandra González-Ospina, Andrea Rodríguez-Restrepo, María Mercedes Grajales-Román, Yeraldyn Espinosa-Chirivi, Marta L. Martínez-Gómez, Andrés A. Agudelo-Suárez

\section{3}

\section{Resumo}

Introdução: para estabelecer sistemas de apoio social para a população universitária, é necessário caracterizá-la em saúde, estilos de vida e práticas de risco.

Objetivo: determinar a frequência e fatores associados com o consumo de álcool, cigarro e substâncias psicoativas em universitários da Faculdade de Odontologia (Universidad de Antioquia, Colômbia).

Métodos: estudo misto: (1) estudo descritivo transversal com 162 participantes (54,3\% mulheres) mediante entrevista e análise uni-bivariada, com o cálculo de frequências e teste de Qui-quadrado; (2) estudo qualitativo com seis grupos focais, análise narrativa de conteúdo e triangulação de resultados. Foram garantidos os princípios éticos para a pesquisa em saúde.

Resultados: 30 \% manifestaram terem se embriagado nos últimos 30 dias (maior proporção em homens: $40 \%$ e $\mathrm{p}<0,05$ ). A idade média de início de consumo de álcool foi de 15 anos (Rango Interquartílico ric 14,0-16,0). A frequência de consumo de cigarro atual foi $11 \%$ e a experiência (atual/passada) foi de $15 \%$, em ambos os casos maior em homens. 0 consumo de substâncias psicoativas no último ano foi de 22 \%, e a idade média de início do consumo foi de 16 anos (ric 15,0-18,5) (mais precoce entre os homens). A análise qualitativa revelou categorias relacionadas com o consumo: 1) estado anímico, 2) carga acadêmica, 3) utilização do tempo livre, 4) relações interpessoais, 5) condições associadas com apreciações e experiências prévias ante o consumo próprio e de pessoas ao seu redor. Conclusões: fatores associados com o consumo de álcool, cigarro e substâncias psicoativas encontrados devem ser considerados para a elaboração de programas de promoção e prevenção em saúde.

Palavras-chave: consumo de bebidas alcoólicas; hábito de fumar; transtornos relacionados com substâncias psicoativas; estudantes de Odontologia

\section{Introducción}

Durante décadas se han implementado estrategias y políticas para disminuir los índices de consumo de sustancias psicoactivas ilegales o legales en la población mundial [1]. Este es considerado como un problema de salud pública por el daño individual y social que genera en las personas [2] [3]. De igual forma, parece importante analizar los problemas de morbilidad y mortalidad que se encuentran asociados al consumo excesivo y adictivo de estas sustancias, pues se asocia con un mayor riesgo de accidentes de tránsito [4] y exposición a violencia [5] [6]. Dentro de las sustancias adictivas que se pueden mencionar como importantes, están el alcohol [7], el hábito de fumar o tabaquismo [8], y el consumo de otras sustancias psicoactivas ilegales como la marihuana, la cocaína u otras drogas [9] [10].

La población juvenil y, especialmente la universitaria, puede considerarse vulnerable al consumo de diferentes sustancias [7]. Existen factores académicos, sociales, familiares y personales que pueden incidir en su magnitud y severidad [11]. Estos temas han sido abordados en el ámbito internacional y nacional, que se evidencia en estudios que intentan analizar el problema en estudiantes universitarios de diferentes 
4 Frecuencia y factores asociados al consumo de alcohol, cigarrillo y sustancias psicoactivas, en estudiantes de una Universidad Pública en Colombia: un estudio mixto

contextos sociales [7]-[9]. Concretamente en Odontología, al encontrarse dentro del área de la salud, las altas demandas académicas que enfrentan los estudiantes, sumado a situaciones de estrés, ansiedad y mala salud mental [12] [13], junto a otros determinantes, pueden ser factores desencadenantes de consumo de diferentes sustancias y otros hábitos nocivos [14] [15].

La Facultad de Odontología de la Universidad de Antioquia (Medellín, Colombia), es una institución docencia servicio que lleva casi 80 años formando profesionales de la salud bucal. Dos estudios realizados con anterioridad muestran la vulnerabilidad de la población estudiantil a esta dependencia en temas relacionados con salud mental [16] y otros como el consumo de cigarrillo junto a otros actores como personal administrativo y docente [17]. Por tanto, la caracterización del problema en términos epidemiológicos y sociales permite el diseño de estrategias de prevención del consumo, así como identificar aquellos factores protectores que son sujeto de medidas de promoción de la salud y que pueden ser un ejemplo para otras instituciones universitarias del país.

La realización de estudios mediante el uso de múltiples metodologías o estudios mixtos, ha cobrado mayor interés en la agenda científica en el campo odontológico [18] [19]. Esto reconoce el abordaje integral de fenómenos de cierta complejidad que no solamente se circunscriben en términos explicativos y estadísticos, sino que avanzan en la comprensión de las opiniones y representaciones individuales, sociales y contextuales frente a un problema determinado.

Teniendo en cuenta lo anterior, se realizó este estudio con el fin de determinar la frecuencia y los factores asociados al consumo de sustancias psicoactivas, alcohol y cigarrillo en estudiantes de pregrado de la Facultad de Odontología de la Universidad de Antioquia.

\section{Métodos}

Se realizó un estudio mixto [19] con el fin de resaltar ambas corrientes epidemiológicas en el abordaje del fenómeno social que se pretendió investigar. Se utilizó un diseño subsumido en el cual predominó el enfoque cuantitativo, con el fin de caracterizar epidemiológicamente el consumo de alcohol, cigarrillo y sustancias psicoactivas en los estudiantes de la Facultad con relación a algunos factores. Esta información fue complementada con el enfoque cualitativo, el cual permitió comprender e interpretar las experiencias y opiniones frente al consumo y obtener algunas respuestas más allá de los datos cuantitativos. 
Alejandra González-Ospina, Andrea Rodríguez-Restrepo, María Mercedes Grajales-Román, 5 Yeraldyn Espinosa-Chirivi, Marta L. Martínez-Gómez, Andrés A. Agudelo-Suárez

\section{Estudio cuantitativo}

Se trata de un estudio descriptivo de corte transversal en estudiantes de primero a décimo semestre de la Facultad de Odontología de la Universidad de Antioquia. marco muestral se definió de acuerdo con las listas suministradas por la institución en los estudiantes matriculados en los cursos de Sociedad, Salud e Investigación (semestres 1 al 3) y Sociedad, Salud y Servicios (semestres 4 al 10). Para la selección de la población participante, se realizó un muestreo por cuotas, determinando un tamaño muestral inicial de 200 encuestas, con igual distribución por semestre y por sexo. La muestra final fue de 162 personas (54,3\% mujeres), con una tasa de respuesta global de 81\% (hombres: 88\%; mujeres: 74\%).

Se diseñó un formato de encuesta (disponible si se solicita a los autores), con información sociodemográfica, estado de salud, apoyo social y preguntas relacionadas con el consumo de alcohol, cigarrillo y sustancias psicoactivas. Se realizó una prueba piloto en 30 personas (15 hombres) con el fin de revisar la consistencia interna del cuestionario, la comprensión del lenguaje y el tiempo de diligenciamiento. Esto permitió ajustes en el diseño del instrumento final. El trabajo de campo fue realizado entre junio y diciembre de 2016. La encuesta fue autodiligenciada con acompañamiento del grupo de investigación, estableciendo jornadas de sensibilización en cada uno de los semestres académicos, lo que permitió obtener de instrumentos de recolección de calidad para los propósitos del estudio.

Concretamente, las variables utilizadas para el análisis cuantitativo fueron: edad, procedencia (Antioquia/otra zona del país), estrato socioeconómico (según la cuenta de servicios y va de 1 a 6), etapa de formación (de acuerdo con el semestre académico), estado civil, tipo de vivienda, actividad laboral, práctica de deporte, tipo de familia y pertenencia a grupos étnicos minoritarios. Apoyo social (Duke-11): es una escala compuesta de 8 frases que se puntúan según 5 categorías de escala de Likert, que van desde «mucho menos de lo que deseo " (que puntúa 1) a «tanto como deseo " (que puntúa 5). Para obtener las puntuaciones de la escala se suman los valores obtenidos a cada una de las frases y el rango de puntuación oscila entre 11 y 55 puntos, a mayor puntaje mayor apoyo percibido. Se optó por un punto de corte en una puntuación < 32, para considerar el apoyo social percibido como bajo.

Como variables de salud se consideraron: salud general, evaluada mediante la pregunta "en los últimos 12 meses, ¿cómo considera su estado de salud?" Con 5 alternativas de respuesta, y fue dicotomizada como "bueno" (opciones: bueno y muy bueno) y "malo" (opciones: regular, malo y muy malo); salud mental, se midió con el General Health Questionnaire de 12 preguntas (GHQ-12), que es un cuestionario con la finalidad de realizar un tamiz de los trastornos mentales actuales. Se pregunta en 
6 Frecuencia y factores asociados al consumo de alcohol, cigarrillo y sustancias psicoactivas, en estudiantes de una Universidad Pública en Colombia: un estudio mixto

forma de escala de Likert de 4 categorías si la persona ha experimentado recientemente un síntoma en particular. A las 4 categorías de respuesta se les adjudicó el valor 0 (respuestas 1 y 2) o 1 (respuestas 3 y 4). Se suman los puntajes y se consideró como mala salud mental un punto de corte $\geq 3$, según otros estudios en poblaciones de contextos similares.

\section{Estudio cualitativo}

Se trata de un estudio cualitativo descriptivo, que permitió complementar los resultados cuantitativos observados. Para ello, se realizaron 6 grupos focales segmentados por sexo, definidos mediante muestreo teórico, realizando 2 grupos focales por cada etapa de formación (etapa 1: semestres 1-3; etapa 2: semestres 4-7, etapa 3: semestres 8-10). Se estableció un diálogo con los participantes mediante preguntas relacionadas con el ámbito académico y social y las posibles explicaciones para el consumo de alcohol, cigarrillo y sustancias psicoactivas desde las experiencias de los estudiantes sobre sí mismos y sobre personas cercanas a su contexto.

El trabajo de campo cualitativo se llevó a cabo entre julio y diciembre de 2016. Los grupos focales tuvieron una duración entre 20 y 30 minutos. Se contó con un moderador y un observador, lo cual permitió tomar notas sobre el desarrollo de los grupos, como estrategia complementaria para el análisis. Los grupos focales fueron grabados y transcritos textualmente y se realizó análisis narrativo de contenido, de los significados de los discursos. Se realizó análisis manual, no usando ningún software de apoyo.

\section{Aspectos éticos}

Se garantizó que esta investigación cumpliera con los principios éticos según la legislación internacional y nacional para investigación en seres humanos [20] [21]. Se considera de riesgo mínimo en tanto no se interfieren en variables físicas o psicológicas en los estudiantes que participaron. Se guardó confidencialidad y anonimato en el manejo de los datos recolectados y se contó con el consentimiento informado escrito para la realización de las encuestas y los grupos focales. Esta investigación fue aprobada como ejercicio académico en el pregrado de Odontología de las 4 primeras autoras. 
Alejandra González-Ospina, Andrea Rodríguez-Restrepo, María Mercedes Grajales-Román, 7 Yeraldyn Espinosa-Chirivi, Marta L. Martínez-Gómez, Andrés A. Agudelo-Suárez

\section{Resultados}

\section{Análisis cuantitativo}

Las variables sociodemográficas y de salud se muestran en las tablas 1 y 2. La mayoría procedían de Antioquia, de estratos medios y bajos, con estado civil soltero. Un poco más de la mitad viven en viviendas propias, una décima parte de la población encuestada trabaja y una buena parte no practican deporte (especialmente las mujeres). Tres cuartas partes de la muestra seleccionada procedían de familias nucleares y en su gran mayoría no reportaron pertenecer a algún grupo étnico minoritario. Un poco menos de la décima parte de la población reportó su apoyo social como bajo. Casi una quinta parte de la muestra reportó su salud general como mala (porcentaje un poco mayor en las mujeres). Más de la mitad reportaron su salud mental como mala (mayor porcentaje en las mujeres). Un 22\% de los hombres y un $14 \%$ de las mujeres reportaron sobrepeso y obesidad (diferencias estadísticamente significativas $\mathrm{p}<0,05)$.

Tabla 1. Características sociodemográficas de la población de estudio.

\begin{tabular}{|c|c|c|c|c|c|c|}
\hline \multirow{2}{*}{ Variables } & \multicolumn{2}{|c|}{ Hombres } & \multicolumn{2}{|c|}{ Mujeres } & \multicolumn{2}{|c|}{ Total } \\
\hline & $\mathbf{n}$ & $\%$ & $\mathbf{n}$ & $\%$ & $\mathbf{n}$ & $\%$ \\
\hline \multicolumn{7}{|l|}{ Edad (años cumplidos) } \\
\hline$\leq 22$ & 34 & 45,9 & 46 & 52,9 & 80 & 49,7 \\
\hline 23 y más & 40 & 54,1 & 41 & 47,1 & 81 & 50,3 \\
\hline \multicolumn{7}{|l|}{ Procedencia } \\
\hline Antioquia & 59 & 79,7 & 75 & 85,2 & 134 & 82,7 \\
\hline Otra zona del país & 15 & 20,3 & 13 & 14,8 & 28 & 17,3 \\
\hline \multicolumn{7}{|l|}{ Estrato socioeconómico } \\
\hline Bajo (1-2) & 20 & 27,0 & 25 & 28,4 & 45 & 27,8 \\
\hline Medio (3-4) & 50 & 67,6 & 61 & 69,3 & 111 & 68,5 \\
\hline Alto (5-6) & 4 & 5,4 & 2 & 2,3 & 6 & 3,7 \\
\hline \multicolumn{7}{|l|}{ Etapa de formación } \\
\hline I (semestres 1 al 3) & 25 & 33,8 & 28 & 31,8 & 53 & 32,7 \\
\hline II (semestres 4 al 7) & 20 & 27,0 & 32 & 36,4 & 52 & 32,1 \\
\hline III (semestres 8 al 10) & 29 & 39,2 & 28 & 31,8 & 57 & 35,2 \\
\hline \multicolumn{7}{|l|}{ Estado Civil } \\
\hline Soltero & 72 & 97,3 & 79 & 89,8 & 151 & 93,2 \\
\hline Casado-Unión en libre & 2 & 2,7 & 6 & 6,8 & 8 & 5,0 \\
\hline Separado & 0 & 0,0 & 3 & 3,4 & 3 & 1,9 \\
\hline
\end{tabular}


8 Frecuencia y factores asociados al consumo de alcohol, cigarrillo y sustancias psicoactivas, en estudiantes de una Universidad Pública en Colombia: un estudio mixto

(viene)

\begin{tabular}{|c|c|c|c|c|c|c|}
\hline \multirow{2}{*}{ Variables } & \multicolumn{2}{|c|}{ Hombres } & \multicolumn{2}{|c|}{ Mujeres } & \multicolumn{2}{|c|}{ Total } \\
\hline & $\mathbf{n}$ & $\%$ & $\mathbf{n}$ & $\%$ & $\mathbf{n}$ & $\%$ \\
\hline \multicolumn{7}{|l|}{ Tipo de vivienda } \\
\hline Propia & 49 & 66,2 & 48 & 54,5 & 97 & 59,9 \\
\hline Arrendada- Otra & 25 & 33,8 & 40 & 45,4 & 65 & 40,1 \\
\hline \multicolumn{7}{|l|}{ Trabaja } \\
\hline Si & 9 & 12,2 & 8 & 9,1 & 17 & 10,5 \\
\hline No & 65 & 87,8 & 80 & 90,9 & 145 & 89,5 \\
\hline \multicolumn{7}{|l|}{ Practica deporte } \\
\hline $\mathrm{Si}$ & 43 & 58,1 & 23 & 26,1 & 66 & 40,7 \\
\hline No & 31 & 41,9 & 65 & 73,9 & 96 & 59,3 \\
\hline \multicolumn{7}{|l|}{ Tipología familiar } \\
\hline Nuclear & 55 & 77,5 & 63 & 73,3 & 118 & 75,2 \\
\hline Monoparental & 9 & 12,7 & 13 & 15,1 & 22 & 14,0 \\
\hline Extensa-Ensamblada & 7 & 9,9 & 10 & 11,7 & 17 & 10,8 \\
\hline \multicolumn{7}{|c|}{ Grupo étnico (pertenencia) } \\
\hline Si & 1 & 1,4 & 8 & 9,1 & 9 & 5,6 \\
\hline No & 73 & 98,6 & 80 & 90,9 & 153 & 94,4 \\
\hline Total & 74 & 45,7 & 88 & 54,3 & 162 & 100,0 \\
\hline
\end{tabular}

Valores perdidos: edad $n=1$, tipo de familia $n=5$.

Fuente: elaboración propia.

Tabla 2. Apoyo social y variables de salud en la población de estudio.

\begin{tabular}{|c|c|c|c|c|c|c|c|}
\hline \multirow{2}{*}{ Variables } & \multicolumn{2}{|c|}{ Hombres } & \multicolumn{2}{|c|}{ Mujeres } & \multirow{2}{*}{ P-valor } & \multicolumn{2}{|c|}{ Total } \\
\hline & $\mathbf{n}$ & $\%$ & $\mathbf{n}$ & $\%$ & & $\mathbf{n}$ & $\%$ \\
\hline \multicolumn{8}{|l|}{ Apoyo social (Duke 11) } \\
\hline Normal & 68 & 91,9 & 80 & 90,9 & \multirow{2}{*}{0,824} & 148 & 91,4 \\
\hline Bajo & 6 & 8,1 & 8 & 9,1 & & 14 & 8,6 \\
\hline \multicolumn{8}{|l|}{ Salud general autopercibida } \\
\hline Buena & 58 & 85,3 & 66 & 79,5 & \multirow{2}{*}{0,357} & 124 & 82,1 \\
\hline Mala & 10 & 14,7 & 17 & 20,5 & & 27 & 17,9 \\
\hline \multicolumn{8}{|l|}{ Salud Mental (GHQ-12) } \\
\hline Buena & 39 & 52,7 & 34 & 38,6 & \multirow{2}{*}{0,073} & 73 & 45,1 \\
\hline Mala & 35 & 47,3 & 54 & 61,4 & & 89 & 54,9 \\
\hline \multicolumn{8}{|c|}{ Indice de Masa Corporal (autopercibido) } \\
\hline Normal & 58 & 78,4 & 70 & 79,5 & \multirow{3}{*}{0,038} & 128 & 79,0 \\
\hline Infrapeso & 0 & 0,0 & 6 & 6,8 & & 6 & 3,7 \\
\hline Sobrepeso-Obesidad & 16 & 21,6 & 12 & 13,6 & & 28 & 17,3 \\
\hline
\end{tabular}

Valores perdidos: salud general autopercibida $n=11$

Fuente: elaboración propia. 
Alejandra González-Ospina, Andrea Rodríguez-Restrepo, María Mercedes Grajales-Román, Yeraldyn Espinosa-Chirivi, Marta L. Martínez-Gómez, Andrés A. Agudelo-Suárez

\section{.}

En la figura 1, se presentan las frecuencias de consumo de alcohol, cigarrillo y otras sustancias psicoactivas en la población estudiantil universitaria participante del estudio. Un 39\% de los hombres reportó haberse embriagado en los últimos 30 días, frente a un $21 \%$ de las mujeres $(p<0,05)$. Un porcentaje mayor al $70 \%$ reportó haberse embriagado al menos en una ocasión (porcentaje superado por los hombres con diferencias estadísticamente significativas ( $p<0,01$ ). Más del 16\% de los hombres reportó fumar actualmente y un 24\% reportó haber fumado (porcentajes más altos frente a las mujeres y con diferencias estadísticamente significativas). Un 15\% de los hombres reportó consumo actual de sustancias psicoactivas y casi una tercera parte reportó consumo en el último año (diferencias estadísticamente significativas frente a las mujeres). La edad mediana de inicio de consumo de alcohol se situó en 15 años para hombres y mujeres, y de sustancias psicoactivas fue de 16 años para hombres y de 18 años para mujeres (figura 2).

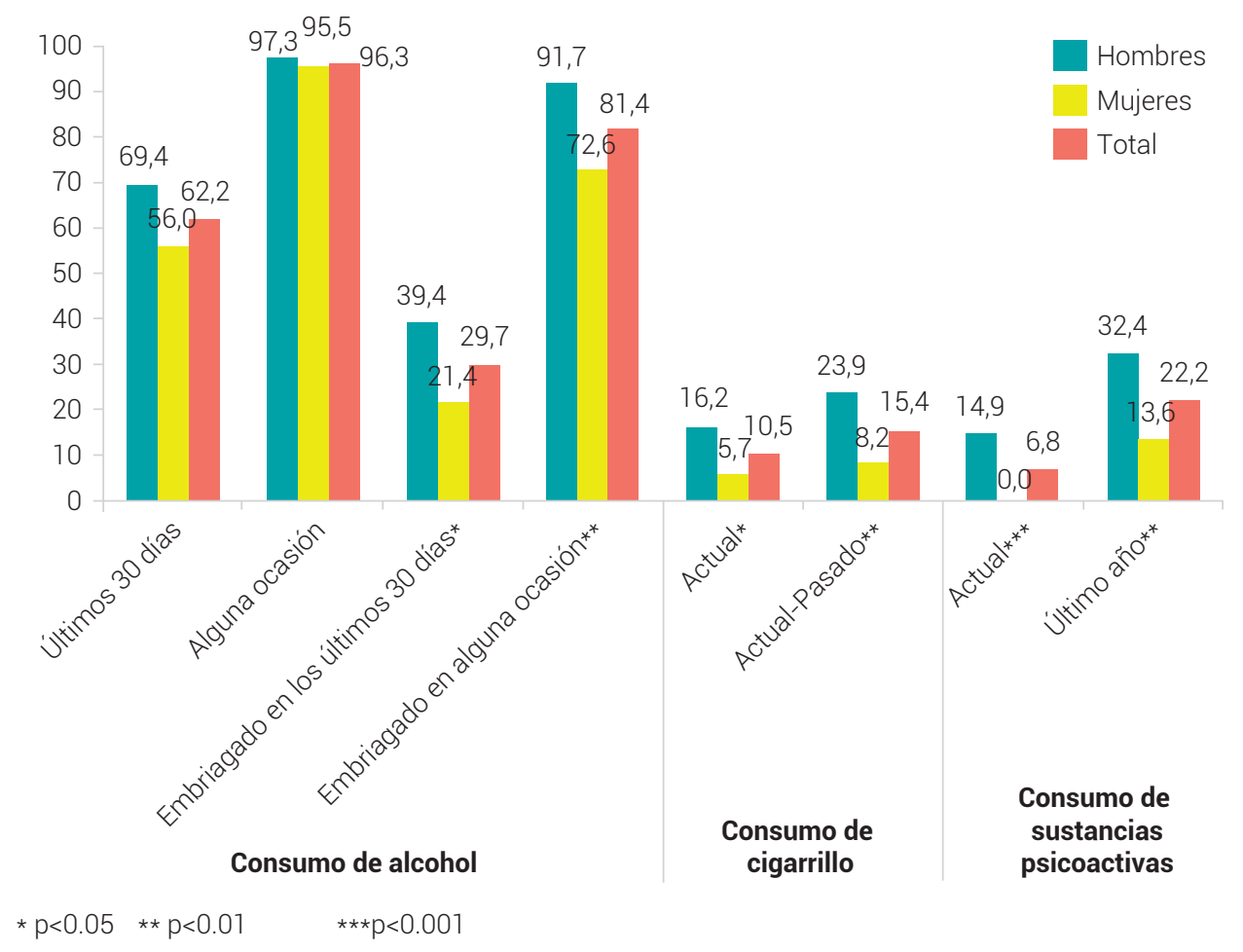

Figura 1. Consumo de alcohol, cigarrillo y otras sustancias psicoactivas en la población participante del estudio.

Fuente: elaboración propia. 


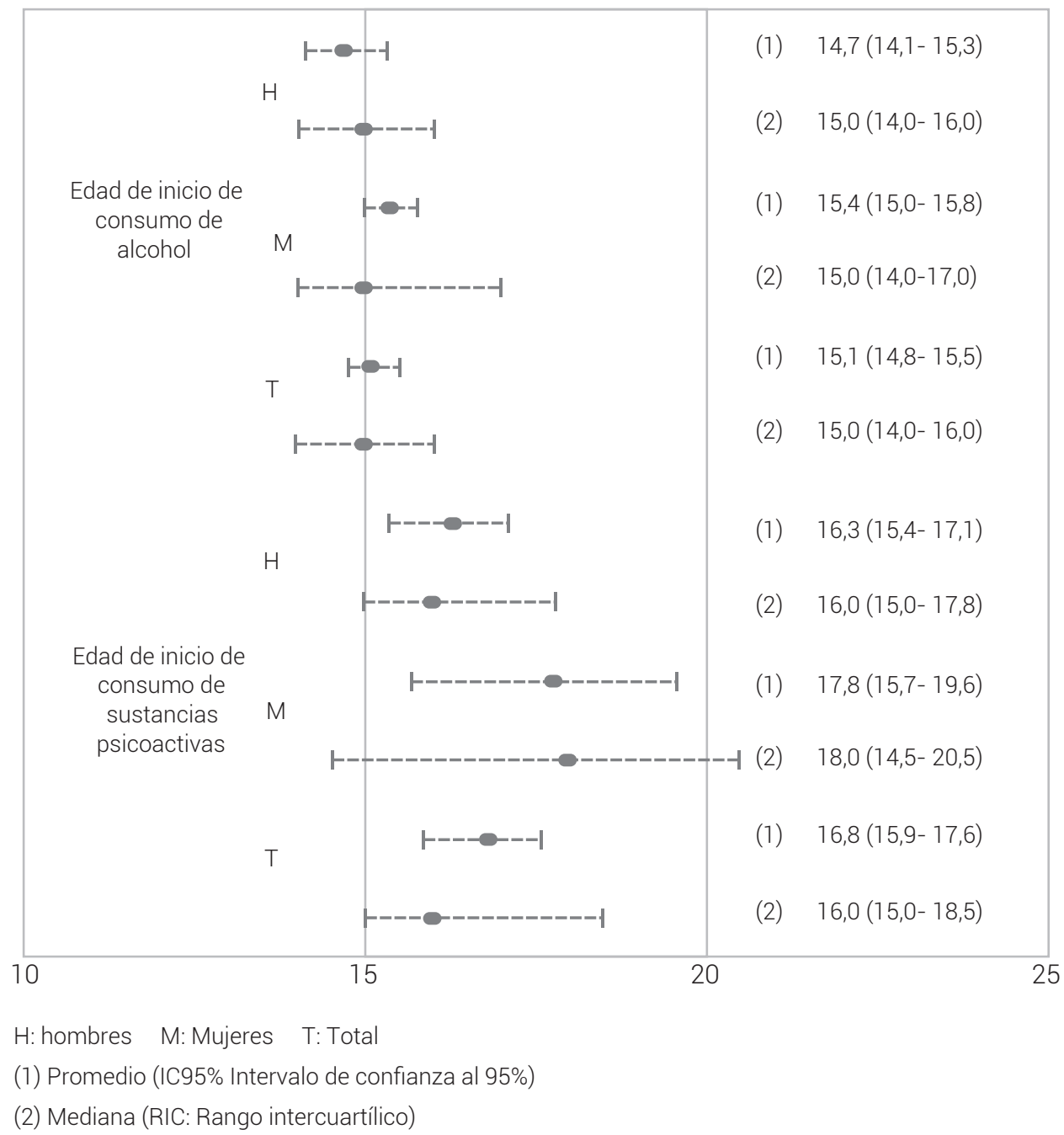

Figura 2. Promedio y mediana de edad en el consumo de alcohol y sustancias psicoactivas en la población de estudio.

Fuente: elaboración propia.

En la tabla 3 se presentan las frecuencias de consumo de alcohol, cigarrillo y otras sustancias psicoactivas según variables sociodemográficas y de salud. En términos generales, no se presentaron diferencias sustanciales en el consumo cuando se analizan las variables seleccionadas, aunque sí se observan pequeñas diferencias por sexo y algunas excepciones. A manera de ejemplo, el consumo fue más alto en estratos socioeconómicos medio y alto para las tres sustancias consideradas. El consumo de cigarrillo fue más alto en los últimos semestres y en personas que trabajan. El consumo de sustancias psicoactivas fue más alto en personas que reportan bajo apoyo social y mala salud mental. El consumo de cigarrillo fue más alto en hombres 
Alejandra González-Ospina, Andrea Rodríguez-Restrepo, María Mercedes Grajales-Román, Yeraldyn Espinosa-Chirivi, Marta L. Martínez-Gómez, Andrés A. Agudelo-Suárez

que reportan sobrepeso y obesidad y para el caso de las mujeres fue más alto en relación con esta misma variable del IMC, pero según el consumo de sustancias psicoactivas.

Tabla 3. Consumo de alcohol, cigarrillo y otras sustancias psicoactivas en la población participante del estudio según variables sociodemográficas y de salud.

\begin{tabular}{|c|c|c|c|c|c|c|c|c|c|c|c|c|}
\hline \multirow{3}{*}{ Variables } & \multicolumn{4}{|c|}{$\begin{array}{l}\text { Embriagado en los últimos } \\
30 \text { días (Si) }\end{array}$} & \multicolumn{4}{|c|}{$\begin{array}{c}\text { Consumo de cigarrillo } \\
\text { actual (Si) }\end{array}$} & \multicolumn{4}{|c|}{$\begin{array}{l}\text { Consumo de sustancias } \\
\text { psicoactivas en el último año ( } \mathrm{Si}\end{array}$} \\
\hline & \multicolumn{2}{|c|}{ Hombres } & \multicolumn{2}{|c|}{ Mujeres } & \multicolumn{2}{|c|}{ Hombres } & \multicolumn{2}{|c|}{ Mujeres } & \multicolumn{2}{|c|}{ Hombres } & \multicolumn{2}{|c|}{ Mujeres } \\
\hline & $\mathbf{n}$ & $\%$ & $\mathbf{n}$ & $\%$ & $\mathbf{n}$ & $\%$ & $\mathbf{n}$ & $\%$ & $\mathbf{n}$ & $\%$ & $\mathbf{n}$ & $\%$ \\
\hline \multicolumn{13}{|c|}{ Edad (años cumplidos) } \\
\hline$\leq 22$ & 11 & 34,4 & 6 & 13,3 & 5 & 14,7 & 0 & $0,0^{*}$ & 15 & $44,1^{*}$ & 8 & 17,4 \\
\hline 23 y más & 17 & 43,6 & 12 & 31,6 & 7 & 17,5 & 5 & $12,2^{*}$ & 9 & $22,5^{*}$ & 4 & 9,8 \\
\hline \multicolumn{13}{|l|}{ Procedencia } \\
\hline Antioquia & 25 & 43,1 & 15 & 21,1 & 9 & 15,3 & 5 & 6,7 & 19 & 32,2 & 10 & 13,3 \\
\hline Otra zona del país & 3 & 23,1 & 3 & 23,1 & 3 & 20,0 & 0 & 0,0 & 5 & 33,3 & 2 & 15,4 \\
\hline \multicolumn{13}{|c|}{ Estrato socioeconómico } \\
\hline Bajo (1-2) & 3 & $15,8^{*}$ & 2 & 8,3 & 1 & 5,0 & 1 & 4,0 & 5 & 25,0 & 2 & 8,0 \\
\hline Medio-Alto (3-6) & 25 & $48,1^{*}$ & 16 & 26,7 & 11 & 20,4 & 4 & 6,3 & 19 & 35,2 & 10 & 15,9 \\
\hline \multicolumn{13}{|l|}{ Etapa de formación } \\
\hline I (semestres 1 al 3) & 8 & 34,8 & 2 & $8,0^{*}$ & 3 & 12,0 & 0 & 0,0 & 9 & 36,0 & 5 & 17,9 \\
\hline II (semestres 4 al 7) & 8 & 40,0 & 6 & $18,8^{*}$ & 4 & 20,0 & 2 & 6,3 & 8 & 40,0 & 4 & 12,5 \\
\hline $\begin{array}{l}\text { III (semestres } 8 \\
\text { al 10) }\end{array}$ & 12 & 42,9 & 10 & $37,0^{*}$ & 5 & 17,5 & 3 & 10,7 & 7 & 24,1 & 3 & 10,7 \\
\hline \multicolumn{13}{|l|}{ Tipo de vivienda } \\
\hline Propia & 20 & 43,5 & 9 & 20,0 & 8 & 16,3 & 4 & 8,3 & 17 & 34,7 & 6 & 12,5 \\
\hline Arrendada- Otra & 8 & 32,0 & 9 & 23,1 & 4 & 16,0 & 1 & 2,5 & 7 & 28,0 & 6 & 15,0 \\
\hline \multicolumn{13}{|l|}{ Trabaja } \\
\hline $\mathrm{Si}$ & 5 & 55,6 & 3 & 37,5 & 1 & 11,1 & 1 & 12,5 & 3 & 33,3 & 1 & 12,5 \\
\hline No & 23 & 37,1 & 15 & 19,7 & 11 & 16,9 & 4 & 5,0 & 21 & 32,3 & 11 & 13,8 \\
\hline \multicolumn{13}{|l|}{ Practica deporte } \\
\hline $\mathrm{Si}$ & 17 & 40,5 & 6 & 26,1 & 6 & 14,0 & 1 & 4,3 & 16 & 37,2 & 2 & 8,7 \\
\hline No & 11 & 37,9 & 12 & 19,7 & 6 & 19,4 & 4 & 6,2 & 8 & 25,8 & 10 & 15,4 \\
\hline \multicolumn{13}{|l|}{ Tipología familiar } \\
\hline Nuclear & 21 & 39,6 & 12 & 20,3 & 7 & 12,7 & 3 & 4,8 & 17 & 30,9 & 6 & 9,5 \\
\hline Otras & 6 & 40,0 & 5 & 21,7 & 4 & 25,0 & 2 & 8,7 & 6 & 37,5 & 4 & 17,4 \\
\hline \multicolumn{13}{|c|}{ Apoyo social (Duke-11) } \\
\hline Normal & 27 & 41,5 & 14 & 18,4 & 10 & 14,7 & 4 & 5,0 & 20 & 29,4 & 10 & 12,5 \\
\hline Bajo & 1 & 16,7 & 4 & 50,0 & 2 & 33,3 & 1 & 12,5 & 4 & 66,7 & 2 & 25,0 \\
\hline \multicolumn{13}{|c|}{ Salud general autopercibida } \\
\hline Buena & 22 & 40,0 & 13 & 20,6 & 9 & 15,5 & 2 & $3,0^{*}$ & 17 & $29,3^{*}$ & 11 & 16,7 \\
\hline Mala & 5 & 50,0 & 4 & 23,5 & 2 & 20 & 3 & $17,6^{*}$ & 6 & $60,0^{*}$ & 1 & 5,9 \\
\hline
\end{tabular}




\begin{tabular}{|c|c|c|c|c|c|c|c|c|c|c|c|c|}
\hline \multirow{3}{*}{ Variables } & \multicolumn{4}{|c|}{$\begin{array}{l}\text { Embriagado en los últimos } \\
30 \text { días ( } \mathrm{Si})\end{array}$} & \multicolumn{4}{|c|}{$\begin{array}{c}\text { Consumo de cigarrillo } \\
\text { actual (Si) }\end{array}$} & \multicolumn{4}{|c|}{$\begin{array}{c}\text { Consumo de sustancias } \\
\text { psicoactivas en el último año (Si) }\end{array}$} \\
\hline & \multicolumn{2}{|c|}{ Hombres } & \multicolumn{2}{|c|}{ Mujeres } & \multicolumn{2}{|c|}{ Hombres } & \multicolumn{2}{|c|}{ Mujeres } & \multicolumn{2}{|c|}{ Hombres } & \multicolumn{2}{|c|}{ Mujeres } \\
\hline & $n$ & $\%$ & n & $\%$ & $\mathbf{n}$ & $\%$ & n & $\%$ & $\mathbf{n}$ & $\%$ & n & $\%$ \\
\hline \multicolumn{13}{|c|}{ Salud Mental (GHQ-12) } \\
\hline Buena & 15 & 39,5 & 6 & 18,8 & 5 & 12,8 & 1 & 2,9 & 10 & 25,6 & 4 & 11,8 \\
\hline Mala & 13 & 39,4 & 12 & 23,1 & 7 & 20,0 & 4 & 7,4 & 14 & 40,0 & 8 & 14,8 \\
\hline \multicolumn{13}{|c|}{ Índice de Masa Corporal (autopercibido) } \\
\hline Normal & 21 & 37,5 & 16 & 24,2 & 10 & 17,2 & 5 & 7,1 & 21 & 36,2 & 9 & 12,9 \\
\hline Infrapeso & 0 & 0,0 & 0 & 0,0 & 0 & 0,0 & 0 & 0,0 & 0 & 0,0 & 0 & 0,0 \\
\hline $\begin{array}{l}\text { Sobrepeso- } \\
\text { Obesidad }\end{array}$ & 7 & 46,7 & 2 & 16,7 & 2 & 12,5 & 0 & 0,0 & 3 & 18,8 & 3 & 25,0 \\
\hline
\end{tabular}

Valores perdidos: te has embriagado en los últimos 30 días $n=8$

${ }^{*} p<0,05$

Fuente: elaboración propia.

\section{Análisis cualitativo}

El análisis cualitativo permitió reconocer los factores asociados al consumo de alcohol, cigarrillo y sustancias psicoactivas en los estudiantes de pregrado participantes del estudio. Estos se describen a continuación según los discursos encontrados en los grupos focales:

- Estado anímico: según las experiencias relatadas por la población entrevistada, dependiendo del estado anímico en el que se encontraban variaba la frecuencia de consumo, siendo mayor en los estados en que se expresan mayor actividad académica o en los momentos de ocio.

"... hay veces que la gente no está en sus días, que tiene muchos problemas o algo y esto puede propiciar mucho esto" (GF 3)

"Yo sí bebo, pues una polita, unas cervecitas, o mediecita de guaro o de tequilita, pues depende del ánimo y el grupo de personas" (GF 1)

- Carga académica: en los discursos de la población entrevistada se puede identificar que a medida que avanzan los semestres la carga académica va aumentando, siendo mayor en la última etapa de la carrera que va de octavo a décimo, lo que puede ser influenciado por los niveles de estrés que presenta cada estudiante.

"podría decirse que influencia la carga académica y todo esto, pero es si usted quiere" (GF 3) 
Alejandra González-Ospina, Andrea Rodríguez-Restrepo, María Mercedes Grajales-Román,

Yeraldyn Espinosa-Chirivi, Marta L. Martínez-Gómez, Andrés A. Agudelo-Suárez

"a mí personalmente el estrés de la universidad no me influye ni en el cigarrillo ni en ninguna otra cosa..." (GF 3)

"... porque uno puede decir estoy mamado el viernes, no quiero más odontología, tomémonos una cerveza" (GF 5)

- Tiempo libre: este factor se ve afectado conforme a la carga académica, ya que a mayor carga académica menor tiempo libre. De acuerdo con las entrevistas se encuentra que este es un factor subjetivo, ya que depende de la organización del tiempo de cada persona.

"La carga académica depende mucho de que uno se organice los tiempos..." (GF 1)

"No porque el poquito tiempo que uno cree que va a tener libre lo tiene que invertir haciendo cosas de la universidad, entonces no he tenido tiempo libre" (GF 5)

- Relaciones interpersonales: se puede observar que estas pueden influir en el consumo, teniendo en cuenta los vínculos de amistad y los diferentes escenarios donde comparten.

"...son personas como que antes nunca lo han hecho y van y lo intentan porque están con un grupo de amigos y van y experimentan" (GF 1)

"O sea, si porque uno comienza y se relaciona con gente del salón, avanza y se relaciona con gente de otros semestres, eso también influiría" (GF 1)

- Condiciones relacionadas con apreciaciones y experiencias previas: este fue otro de los factores que se encontró en los grupos focales, en los que se relacionaba el consumo actual con los juicios morales emitidos por la sociedad, en donde las sustancias legales eran vistas como "buenas" y se aprueba su consumo; a diferencia de las sustancias ilegales. También se encontró que había personas que iniciaban este consumo antes de iniciar la universidad guiados por la curiosidad de conocer los efectos que producen estas sustancias.

"Yo sí he probado, pues no lo hice ni porque me obligaron ni por nada y pues simplemente era algo que quería experimentar en mi vida" (GF 1)

"...dicen que no es bueno para la salud, pero eso lo elige cada persona, entonces yo la verdad lo veo muy normal..." (GF 5)

\section{Discusión}

Los hallazgos principales de este estudio dan cuenta de que existe un consumo de cigarrillo, alcohol y sustancias psicoactivas entre moderado y alto, que varía según 
el tipo de sustancia, el periodo de consumo y las diferentes variables sociodemográficas y de salud consideradas en el análisis. El inicio para el consumo de sustancias psicoactivas y alcohol se da durante el periodo de la adolescencia y con diferencias según sexo (inicio más temprano en los hombres). La población entrevistada en los grupos focales, manifestó factores relacionados con el ambiente académico y sus propias experiencias personales y de grupo que pueden influir en el consumo de las diferentes sustancias consideradas en el estudio. Hasta donde alcanza nuestro conocimiento, es uno de los primeros estudios que pretende caracterizar y comprender este fenómeno social en estudiantes de odontología de la ciudad.

En la comparación de los resultados de este estudio es necesario tener en cuenta algunas consideraciones, ya que los diferentes estudios en población universitaria, utilizan diferentes instrumentos para medir el consumo y tienen diferentes formas de operacionalizar de las diferentes variables. Concretamente, en estudiantes de Odontología, las prevalencias de consumo de cigarrillo encontradas en el estudio colombiano, son inferiores a las reportadas en un estudio realizado en el Reino Unido [15]. Aunque este mismo estudio reporta altos consumos de alcohol de forma ocasional (86\% para hombres y $84 \%$ para mujeres) o excesiva (35\% hombres y $41 \%$ mujeres). En este último caso, llama la atención el reporte de mayor consumo en mujeres frente al estudio local, el cual reporta consumos más altos para hombres. El consumo de sustancias psicoactivas fue más bajo en comparación al consumo local [15]. Otra investigación de carácter longitudinal realizada en el mismo contexto europeo muestra, por ejemplo, que el consumo de alcohol en niveles que se consideran peligrosos aumenta a medida que avanzan las etapas de formación académica, continuando este patrón de consumo como profesionales [13]. Una situación parecida se observó con el consumo de sustancias psicoactivas consideradas como ilegales (especialmente marihuana) [13].

Teniendo en cuenta los resultados cualitativos, parece importante analizar los factores socioculturales y las diferentes motivaciones que están relacionadas con el patrón de consumo en estudiantes universitarios. Un estudio realizado con estudiantes de seis universidades colombianas muestra que "sentirse mejor, disminuir la tensión, la ansiedad o el estrés y experimentar nuevas sensaciones", son motivos para el consumo de sustancias psicoactivas [10]. La carga académica y el menor tiempo libre, fueron elementos resaltados por los estudiantes de Odontología en Medellín. Un estudio en población universitaria de Costa Rica [11], reportó que la condición académica es un factor de riesgo asociado a un mayor consumo de alcohol y cigarrillo. Precisamente, el tema de la sobrecarga académica es un asunto que ha sido asociado no solo con el consumo como tal, sino con la deserción estudiantil [22]. 
Alejandra González-Ospina, Andrea Rodríguez-Restrepo, María Mercedes Grajales-Román, 15 Yeraldyn Espinosa-Chirivi, Marta L. Martínez-Gómez, Andrés A. Agudelo-Suárez

Los diferentes patrones de consumo, pueden convertirse en una forma de sobrellevar el malestar sobre todo en momentos críticos, generando una ruptura del equilibrio en lo cotidiano. De igual forma, existen prácticas culturales asociadas al consumo, donde priman elementos de comportamiento individual, elementos relacionados con las propias decisiones y formas de autocontrol sobre sus hábitos y conductas y factores de interacción social [23]. Esto guarda relación con lo encontrado en las percepciones de los estudiantes de Odontología, al involucrar condiciones asociadas con apreciaciones y experiencias previas frente al consumo, los juicios de valor que se establecen y la percepción sobre los hábitos vistos en otras personas.

Si bien no se encontraron diferencias estadísticamente significativas y no fueron consistentes en todos los casos, este estudio evidenció algunas relaciones entre el consumo de alcohol, cigarrillo y otras sustancias con la presencia de indicadores negativos de salud. Cabe anotar que un estudio previo realizado con estudiantes de odontología de la misma universidad, reportó que la prevalencia de síntomas sugerentes de estrés, ansiedad y mala salud general y mental oscila entre el 34 y el 52\% [16]. Un estudio realizado con estudiantes de Cartagena (Colombia), analizó los factores que influyen en síntomas sugerentes de mala salud mental, mostrando asociaciones significativas con el consumo de alcohol [12]. El consumo excesivo de estas sustancias, puede generar consecuencias en términos de las expectativas de la población estudiantil, afectando la salud mental por la disonancia entre sus aspiraciones y sus logros, afectando su sentido de vida y dando paso a esta problemática en salud mental, que han sido investigadas en estudiantes que acuden a consulta psicológica [24]. En el otro lado de la balanza, conviene el análisis de los estilos de vida saludables y cómo estos pueden tener relación con el consumo de sustancias. Al respecto, un estudio realizado en Cali, Colombia [25], muestra que existen factores sociodemográficos y académicos que inciden en el aumento de prácticas saludables. Por ejemplo, estar trabajando aumenta significativamente estos hábitos en el consumo de alcohol, cigarrillo y sustancias consideradas ilegales.

En consonancia con lo anterior, se deben analizar los factores demográficos que ejercen cierta influencia en los patrones de consumo de la población estudiantil considerada en el estudio. Aunque la muestra, en términos generales, es más o menos homogénea, lo que contribuyó a que las diferencias significativas no fueran tan evidentes en la edad, el estrato socioeconómico, la condición laboral y el bajo apoyo social. Otros estudios en estudiantes de odontología realizados en otros contextos geográficos no han analizado en profundidad, factores asociados como los considerados en este estudio [13]-[15]. Esta situación evidencia la necesidad, de estudios de mayor alcance y profundidad, incluyendo estudiantes de Odontología que proceden 
de instituciones públicas y privadas e incluir métodos cualitativos que permitan reconocer la construcción de representaciones y prácticas frente al consumo, y evidenciar hábitos y condiciones generadoras de salud integral que redunden en bienestar para este grupo poblacional.

En la interpretación de los datos y su discusión en el contexto social actual, se hace importante comentar las limitaciones y fortalezas de este estudio. Esta investigación se realizó en un grupo particular de estudiantes de odontología a través de un muestreo no probabilístico y los resultados no deberían replicarse a la población general de universitarios de odontología, aunque debe aclararse que este no era el objetivo del estudio. Se supone, en todo caso, que las condiciones académicas y sociales puedan ser similares en otras universidades públicas del país y esto pone en manifiesto la necesidad de estrategias de intervención y promoción de la salud basadas en la realidad particular que vive la población estudiantil. La utilización de métodos mixtos permitió, por un lado, la utilización de instrumentos de carácter cuantitativo que fueron probadas y estandarizadas previamente y, por otra parte, la identificación y exploración de categorías propias desde la percepción de los participantes del estudio mediante estrategias cualitativas.

Los resultados encontrados evidencian la necesidad de elaborar propuestas de intervención basadas en la realidad social que viven los estudiantes de odontología de la ciudad y del país. Estas estrategias deben reconocer la participación como un elemento que permite identificar las actividades más acordes para promover la salud y prevenir posibles enfermedades y riesgos asociados al consumo excesivo de sustancias nocivas. La vida académica de estos estudiantes ha implicado su desplazamiento a otro espacio y territorio social como es la Universidad. Esto plantea una mayor atención en los servicios de asesoría, acompañamiento y tutoría, así como establecer programas de seguimiento y evaluación de las condiciones sociales y de salud de la población universitaria.

\section{Referencias}

1. Hernández Ramírez EM, Orozco Giraldo IC, Ríos JD. Estado del arte sobre el diseño, análisis y evaluación de políticas de reducción de daño por consumo de sustancias psicoactivas, en Europa y América entre 2003 a 2013. Salud y Drogas. 2017; 17(2): 5-16. Disponible en: https:// ojs.haaj.org/index.php/haaj/article/view/275 
Alejandra González-Ospina, Andrea Rodríguez-Restrepo, María Mercedes Grajales-Román, Yeraldyn Espinosa-Chirivi, Marta L. Martínez-Gómez, Andrés A. Agudelo-Suárez

2. Duffy DN. Consumo de alcohol: principal problemática de salud pública de las Américas. PSIENCIA Revista Latinoamericana de Ciencia Psicológica. 2015; 7(2): 371-382. Disponible en: https://www.redalyc.org/pdf/3331/333141094004.pdf

3. Tirado-Otálvaro AF. El consumo de drogas en el debate de la salud pública. Cad Saúde Pública. 2016; 32(7): e00177215. https://doi.org/10.1590/0102-311X00177215

4. Rodríguez-Guzmán S, Jiménez-Mejías E, Martínez-Ruiz V, Lupiáñez-Tapia F, Lardelli-Claret P, Jiménez-Moleón JJ. Movilidad, accidentalidad por tránsito y sus factores asociados en estudiantes universitarios de Guatemala. Cad Saúde Pública. 2014; 30(4): 735-745. https://doi. org/10.1590/0102-311X00109713

5. Herrera Paredes JM, Ventura CAA. Consumo de alcohol y violencia doméstica contra las mujeres: un estudio con estudiantes universitarias de México. Rev Latino-Am Enfermagem. 2010; 18(Spec): 557-564. Disponible en: https://www.scielo.br/pdf/rlae/v18nspe/a11v18nspe.pdf

6. Saldivia C, Vizcarra B. Consumo de Drogas y violencia en el noviazgo en estudiantes universitarios del sur de Chile. Terapia psicológica. 2012; 30(2): 43-49. http://dx.doi.org/10.4067/ S0718-48082012000200004

7. Betancourth-Zambrano S, Tacán-Bastidas L, Cordoba-Paz EG. Consumo de alcohol en estudiantes universitarios colombianos. Univ Salud.2017;19(1):37-50. http://dx.doi.org/10.22267/ rus. 171901.67

8. Bautista-Pérez F. Prevalencia del consumo de cigarrillos y otras formas de tabaco en estudiantes universitarios. Crea Ciencia Revista Científica. 2014; 9(1): 19-27. Disponible en: https://www.redalyc.org/articulo.oa?id=83940103

9. Patiño-Masó J, Gras-Pérez E, Font-Mayolas S, Baltasar-Bagué A. Consumo de cocaína y policonsumo de sustancias psicoactivas en jóvenes universitarios. Enferm Clin. 2013; 23(2): 62-67. https://doi.org/10.1016/j.enfcli.2013.02.003

10. Duarte Alarcón C, Varela Arévalo MT, Salazar Torres IC, Lema Soto LF, Tamayo Cardona JA. Motivaciones y recursos para el consumo de sustancias psicoactivas en universitarios. Revista Hacia la Promoción de la Salud. 2012; 17(1): 92-104.

11. Caravaca Morera JA, Noh S, Hamilton H, Brands B, Gastaldo D, Miotto Wright MdG. Factores socioculturales y consumo de drogas entre estudiantes universitarios costarricenses. Texto Contexto Enferm.2015;24(Esp):145-153. http://dx.doi.org/10.1590/0104-07072015001170014 
Frecuencia y factores asociados al consumo de alcohol, cigarrillo y sustancias psicoactivas, en estudiantes de una Universidad Pública en Colombia: un estudio mixto

12. Arrieta-Vergara K, Fortich-Mesa N, Tirado-Amador L, Simancas-Pallares M. Common Mental Disorders and Associated Factors in Dental Students From Cartagena, Colombia. Rev Colomb Psiquiatr. 2019; 48(1): 10-6. https://doi.org/10.1016/j.rcpeng.2017.05.016

13. Newbury-Birch D, Lowry R, Kamali F. The Changing Patterns of Drinking, Illicit Drug Use, Stress, Anxiety and Depression in Dental Students in a UK Dental School: a Longitudinal Study. Br Dent J. 2002; 192(11): 646. https://doi.org/10.1038/sj.bdj.4801448

14. Teixeira RF, Souza RSD, Buaiz V, Siqueira MMD. Uso de substâncias psicoativas entre estudantes de odontologia da Universidade Federal do Espírito Santo. Cien Saude Colet. 2010; 15(3): 655-662. https://doi.org/10.1590/S1413-81232010000300007

15. Puryer J, Wignall R. Tobacco, Alcohol and Drug Use among Dental Undergraduates at One UK University in 2015. Dentistry Journal. 2016; 4(1): 2. https://doi.org/10.3390/dj4010002

16. Álvarez-Rúa LJ, Carmona-Rendón YA, Holguín-Vásquez VA, Ortiz-Parra MA, Patiño-Arango SE, Vélez-Peláez S, et al. Salud mental en estudiantes de odontología de una Universidad Pública de Medellín (Colombia) y sus factores relacionados. Rev Nac Odontol. 2019; 15(28): 1-19. https://doi.org/10.16925/2357-4607.2019.02.08

17. Agudelo-Suárez AA, Yepes-Delgado FL, Castro-Naranjo VP, Cano-Restrepo CM, CampuzanoPeláez CM. Consumo de cigarrillo en la Facultad de Odontología de la Universidad de Antioquia y factores relacionados. Medellín, 2012. Rev Fac Odontol Univ Antioq. 2016; 28(2): 261-277.

18. Gupta A, Keuskamp D. Use and Misuse of Mixed Methods in Population Oral Health Research: A Scoping Review. Community Dent Health. 2018; 35(2): 109-118. https://doi.org/10.1922/ CDH_4250Gupta10

19. Nicolaum B, Castonguay G, Levine A, Hong Q, Participants S, Pluye P. Applied Mixed Methods in Oral Health Research: Importance and Example of a Training Program. JDR Clinical \& Translational Research. 2017; 2(3): 206-210. https://doi.org/10.1177/2380084417705823

20. General Assembly of the World Medical A. World Medical Association Declaration of Helsinki: Ethical Principles for Medical Research Involving Human Subjects. J Am Coll Dent. 2014; 81(3): $14-18$.

21. Ministerio de Salud y Protección Social (Colombia). Resolución No. 008430 de 1993 (4 de octubre de 1993). Por la cual se establecen las normas científicas, técnicas y administrativas para la investigación en salud. Bogotá (Colombia): Ministerio de Salud y Protección Social; 1993. 
Alejandra González-Ospina, Andrea Rodríguez-Restrepo, María Mercedes Grajales-Román, 19 Yeraldyn Espinosa-Chirivi, Marta L. Martínez-Gómez, Andrés A. Agudelo-Suárez

22. Delgado FLY, Salazar MB, Ceferino EYM. Academic Dropout Among Undergraduate Students from Universidad de Antioquia School of Dentistry, 2004-2014. Rev Fac Odontol Univ Antioq. 2017; 29(1): 76-95.

23. Novoa Gómez MM, Barreto I, Silva Martín LM. Consumo de cigarrillo y prácticas culturales en contextos universitarios. Rev Latin Psic 2012; 44(1): 97-110.

24. Micin S, Bagladi V. Salud mental en estudiantes universitarios: incidencia de psicopatología y antecedentes de conducta suicida en población que acude a un servicio de salud estudiantil. Terapia Psicológica. 2011;29(1):53-64. http://dx.doi.org/10.4067/S0718-48082011000100006

25. Tamayo Cardona JA, Rodríguez K, Escobar K, Mejía AM. Estilos de vida de estudiantes de odontología. Rev Hacia la Promoción de la Salud. 2015; 20(2): 147-160. 